\title{
Nonlinear temporal Fresnel diffraction induced by phase jumps in fiber optics
}

\author{
Anastasiia Sheveleva and Christophe Finot* \\ Laboratoire Interdisciplinaire Carnot de Bourgogne, UMR 6303, UBFC-CNRS, 9 av. A. Savary, 21000 Dijon, France
}

\begin{abstract}
We numerically study the temporal patterns that emerge upon the propagation of one or two $\pi$ phase jumps in a nonlinear optical fiber. Both normal and anomalous regimes of dispersion are considered. The analytical derivation of linear patterns based on Fresnel's integrals provides some clues to better understand the properties of the resulting coherent structures.
\end{abstract}

\section{Introduction}

Diffraction is among the key effects of wave physics with applications in a broad range of technological fields. The knowledge accumulated in the field of spatial optics over the last two centuries is a valuable help to provide insights to a better understanding of the temporal dynamics of a wave experiencing the dispersive propagation. Indeed, the parabolic spectral phase induced by the dispersion of an ultrashort optical pulse is equivalent to the paraxial diffraction affecting the spatial propagation of a light beam [1]. This has been the basis of various recent applications in ultrafast optics including, for example, a temporal analogue to the Arago's spot demonstrated in optical fibers [2].

However, up to now, most the works have mainly focused to the evolution of fields that have been intensity modulated. We here discuss the temporal evolution of a continuous wave affected by one or two abrupt $\pi$-phase shifts, which is the temporal equivalent of a plane wave transmitted through a transparent plate where a localized phase jump leads to $\lambda / 2$ optical path difference. We are particularly interested in the effects of the Kerr nonlinearity on the propagating wave in both the anomalous and normal regimes. We highlight the emergence of coherent nonlinear structures such as bright solitons, Peregrine solitons or black solitons.

\section{Evolution of a single phase jump}

We first consider the evolution of the wave affected by an ideal single $\pi$-phase shift (Fig. 1, panel a, grey line). The light propagates through a $10-\mathrm{km}$ long anomalously dispersive fiber with parameters typical of the SMF-28 fiber at telecommunication wavelengths. When nonlinearity is neglected, the temporal intensity pattern can be derived analytically as [3] (see panel a, black line):

$$
I(t)=\frac{1}{2}\left[C_{f}^{2}\left(\frac{\operatorname{sgn}\left(\beta_{2}\right) t}{\sqrt{\pi\left|\beta_{2}\right| z}}\right)+S_{f}^{2}\left(\frac{\operatorname{sgn}\left(\beta_{2}\right) t}{\sqrt{\pi\left|\beta_{2}\right| z}}\right)\right]
$$

with $t$ being the temporal coordinate and $C_{f}$ and $S_{f}$ being the Fresnel integrals, $\beta_{2}$ the second-order dispersion and $z$ the propagation distance. This analytical expression can be straightforwardly interpreted by the use of the clothoid. The symmetric pattern that emerges is characterized by a central hole surrounded by two marked oscillating edges that move in opposite directions.
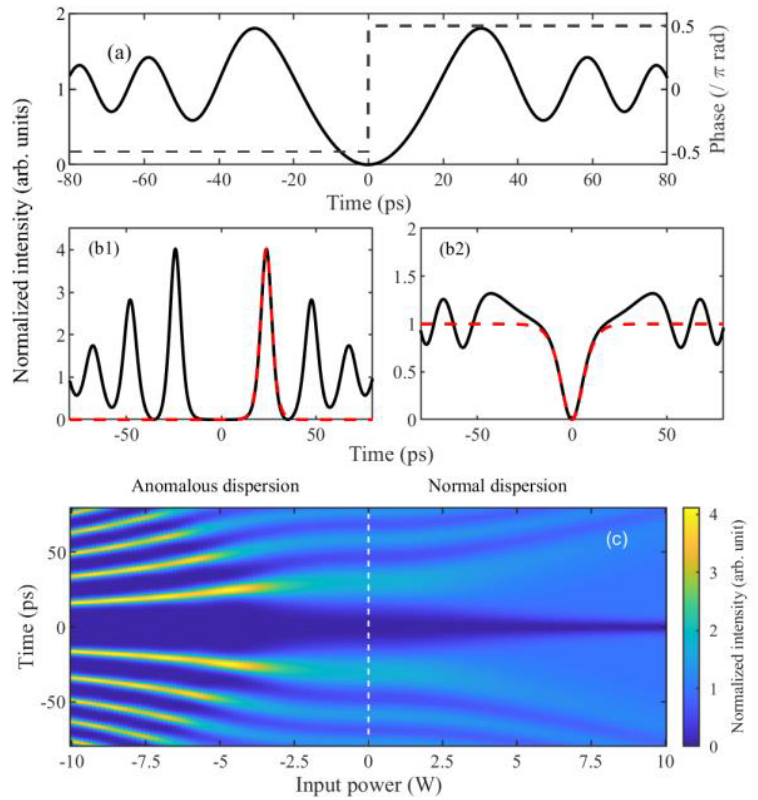

Fig. 1. (a) Initial phase jump (dashed line) and output intensity profile without nonlinearity. (b) Details of the impact of nonlinearity in the anomalous and normal dispersion (b1 and b2). Red dashed lines are fits by a bright and a black soliton. (c) Influence of the initial average power on the output pattern.

When Kerr nonlinearity impacts the propagation, the field evolution is governed by the nonlinear Schrödinger equation that is numerically solved [4]. Output profiles which are plotted on panels (b) stress that two bright fundamental solitons emerge in the anomalous regime of propagation whereas a single black soliton appears in the normal case. When nonlinearity is increased (panel c), the

* Corresponding author: christophe.finot@u-bourgogne.fr 
coherent structures are subjects to the usual scaling laws of solitons.

\section{Evolution of a pair of $\pi$ phase jumps}

We have also numerically considered the evolution of a pair of $\pi$-phase jumps spaced by $\Delta T=40 \mathrm{ps}$. The linear propagation (Fig. 2a) leads to a more complex pattern that can be understood quite easily in terms of interference between the temporal Arago's pattern [5] and the result of the dispersive evolution of a rectangular wave of width $\Delta T$ that is phase shifted by $\pi$. Once again, the linear profile can be analytically expressed in terms of a combination of Fresnel integrals. After $10 \mathrm{~km}$ of linear propagation, the dispersive pattern is made of a broad central peak surrounded by strong ripples which peak intensity has been increased compared to the previous case under discussion.
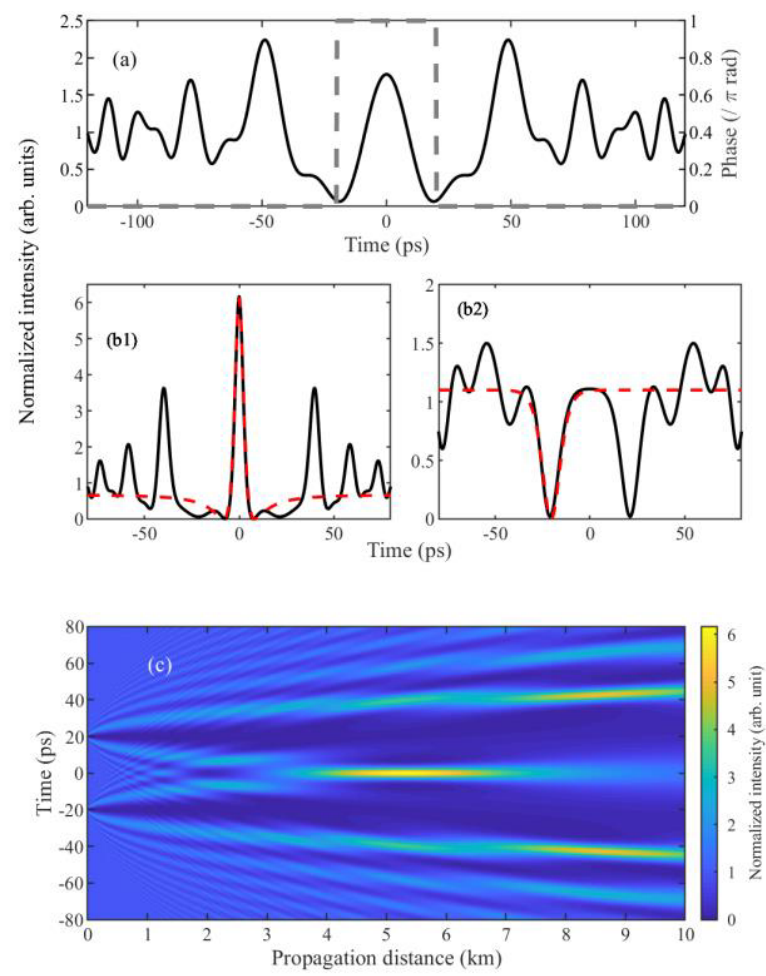

Fig. 2. (a) Initial phase profile (dashed line) and output intensity profile in the linear propagation. (b) Temporal intensity profiles in presence of nonlinearity observed after $5.37 \mathrm{~km}$ in a fiber with anomalous dispersion (b1) or after 10 $\mathrm{km}$ in a fiber with normal dispersion (b2) for an initial power of $290 \mathrm{~mW}$. The red dashed lines are the profiles of a Peregrine soliton at its point of maximum focusing and of a black soliton. (c) Longitudinal evolution of the intensity profile in the anomalous regime of dispersion.

When nonlinearity is experienced, the patterns have very different dynamic according to the regime of dispersion. The anomalous regime is marked by the emergence of a strong central peak that experiences a growth and decay longitudinal evolution (see Fig. 2(c)). At the point of maximum focusing (see Fig. 2(b1)), the ultrashort structure is in close agreement with the shape of a Peregrine soliton [6], with a temporal duration below 6 ps and a peak power that is increased by a factor 6 with respect to the initial average power. This central peak may also split into a doublet of pulses [7] (Fig. 3) and is surrounded by other breathing structures that have also been identified in the nonlinear Fresnel evolution of a supergaussian pulse [8]. These observations confirm the universal impact of coherent structures linked to modulation instability that emerge not only from intensity perturbations, but also from localized phase defects.

In the normal regime of propagation, the picture gets very different. Instead of an intense central structure, the intensity profile becomes nearly constant in-between two black solitons. The position of these dips of light is fixed by position of the initial phase shifts and is not influenced by the power level of the initial continuous wave.

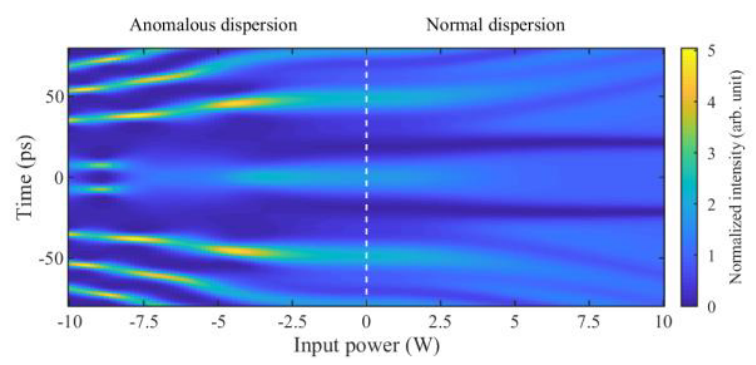

Fig. 3. Influence of the initial average power on the output pattern recorded after $10 \mathrm{~km}$ of propagation

To discuss the influence of the value of the initial phase offset we have carried out additional simulations. The resulting pattern can be significantly different with a loss of the temporal symmetry for the single jump case. Breathing solutions may appear in the anomalous regime of propagation and grey solitons are observed instead of black ones in the normal regime of dispersion. When nonideal phase jumps are considered (i.e. the transition between the two phase states has a given duration), the nonlinear pattern will also be affected by this additional initial chirp.

\section{References}

1. V. Torres-Company, J. Lancis, and P. Andrés, in Progress in Optics, E. Wolf, ed. (Elsevier, 2011), pp. 1-80.

2. C. Finot and H. Rigneault, J. Opt 21, 105504 (2019).

3. H. D. Betz, Appl. Opt. 8, 1007-1013 (1969).

4. G. P. Agrawal, Nonlinear Fiber Optics, Fourth Edition (Academic Press, San Francisco, CA, 2006).

5. J. Komrska, Optica Acta: International Journal of Optics 14, 127-146 (1967).

6. B. Kibler, J. Fatome, C. Finot, G. Millot, F. Dias, G. Genty, N. Akhmediev, and J. M. Dudley, Nat Phys 6, 790-795 (2010).

7. C. Finot, Laser Phys. Lett. 17, 025103 (2020).

8. F. Audo, B. Kibler, J. Fatome, and C. Finot, Opt. Lett. 43, 2864-2867 (2018). 